Article

\title{
Entropy Diagnostics for Fourth Order Partial Differential Equations in Conservation Form
}

\author{
Phil Broadbridge
}

Australian Mathematical Sciences Institute, c/o University of Melbourne, 111 Barry St., Melbourne VIC 3010, Australia

E-mail: phil@amsi.org.au

Received: 3 May 2008 / Accepted: 21 September 2008 / Published: 25 September 2008

\begin{abstract}
The entropy evolution behaviour of a partial differential equation (PDE) in conservation form, may be readily discerned from the sign of the local source term of Shannon information density. This can be easily used as a diagnostic tool to predict smoothing and non-smoothing properties, as well as positivity of solutions with conserved mass. The familiar fourth order diffusion equations arising in applications do not have increasing Shannon entropy. However, we obtain a new class of nonlinear fourth order diffusion equations that do indeed have this property. These equations also exhibit smoothing properties and they maintain positivity. The counter-intuitive behaviour of fourth order diffusion, observed to occur or not occur on an apparently ad hoc basis, can be predicted from an easily calculated entropy production rate. This is uniquely defined only after a technical definition of the irreducible source term of a reaction diffusion equation.
\end{abstract}

Keywords: Shannon entropy, fourth order diffusion, irreversibility

\section{Introduction}

Fourth order diffusion equations arise in many applications such as thin film theory [1, 2], surface diffusion on solids [3-5], interface dynamics [6-8], flow in Hele-Shaw cells, and phase field models of multiphase systems [9]. The simplest prototype is the fourth order diffusion equation with constant coefficients, $u_{t}=-u_{x x x x}$. Although fourth order diffusion equations are dissipative equations, they do not exhibit the smoothing properties usually associated with the very name "diffusion". Unlike second order diffusion equations, they do not obey a robust maximum principle; one cannot rule out the gen- 
eration of new extrema in the evolving solutions of standard initial-boundary problems. Indeed, with fourth order evolution we commonly observe the appearance of new structures evolving from smoother initial conditions (e.g. [1,5] ). Compared to the familiar smoothing behaviour of second order diffusion, the behaviour of some fourth order equations seems counter-intuitive. Rippling effects and smoothing effects occur in a seemingly ad-hoc manner. Although for fourth order equations, there is no robust maximum principle, entropy production rate may be useful as a simple litmus test. The primary purpose of this paper is to demonstrate that the entropy evolution behaviour of a partial differential equation (PDE) in conservation form, may be readily calculated, allowing it to be easily used as a diagnostic tool to predict smoothing and non-smoothing properties, as well as positivity of solutions with conserved mass. The familiar fourth order diffusion equations do not have increasing Shannon entropy. However, we obtain a new class of nonlinear fourth order diffusion equations that do indeed have this property. These equations also exhibit smoothing properties and they maintain positivity.

A secondary outcome is to show that a single scalar transport equation may be used as a simplified prototype of a thermodynamic system, immediately establishing the equivalence between two statements of the second law of thermodynamics, namely equilibrating heat transport and entropy increase. A system described by a single parabolic equation for a single intensive variable is not a thermodynamic system in the usual technical sense. However, the very purpose of the heat equation is to model the dynamics of heat conduction, which is part of thermodynamics in a practical sense. We can use a single scalar equation to illustrate thermodynamic concepts by constructing from it some entropy density $s(x, t)$ that has a non-negative source density that is not strictly zero. The analogue of an adiabatic process is a time evolution of some scalar quantity $T(x, t)$ governed by a parabolic evolution equation, subject to thermal isolation, represented by zero-flux boundary conditions. In this simplified system, the dynamical law for $\mathrm{T}(\mathrm{x}, \mathrm{t})$ is specified even in non-equilibrium conditions. This avoids the complication of temperature not even being uniquely defined in more realistic non-equilibrium systems of many interacting particles.

Although the evolution of standard entropy is rarely used as a tool for the analysis of more general PDEs, we demonstrate here that it is in fact quite useful for this purpose. Before we analyse nonlinear fourth order diffusion equations, we first introduce the basic concepts using the very simplest diffusion equation, namely the classical linear heat equation in 1+1 dimensions. Most of the constructions used here can readily be extended to higher spatial dimensions.

\section{Basic Concepts: The Linear heat Equation}

Consider the classical one-dimensional linear heat equation,

$$
\frac{\partial T}{\partial t}=D \frac{\partial^{2} T}{\partial x^{2}} \quad ; T \in C^{2,1}\left([a, b] \times \Re^{+}\right)
$$

with $-\infty \leq a<b \leq \infty$. For simplicity, we assume that the domain is a fixed region of constant volume and that the enclosed material has uniform composition and uniform density $\rho$. Then we do not need to consider energy transfer due to the work done in compressing the material or in redistributing its components. 
The heat equation first arose as a conservation equation

$$
\frac{\partial q}{\partial t}+\frac{\partial J}{\partial x}=0
$$

with $q=\rho C_{V} T, C_{V}$ being the specific heat, and

$$
J=-k \frac{\partial T}{\partial x} \quad(\text { Fourier's law })
$$

$\mathrm{k}$ being the thermal conductivity, $k=\rho C_{V} D$.

As noted by Lieb and Yngvason [10], it is remarkable that more than 150 years since the definition of entropy, there is no universally agreed formulation of irreversible thermodynamics of macroscopic systems. In the original definition of Clausius, the entropy $\mathrm{S}$ of a closed system is a quantity that has an exact differential $\delta S=\delta Q / T$, where $\delta Q$ is the infinitesimal increase in heat content during an adiabatic process that notionally connects one equilibrium state to another. For a simplified non-equilibrium system that is described by a single scalar heat conservation equation on a rigid domain with insulated boundary, there can be no change in the total heat content. However, heat will still flow irreversibly from one open subregion to another. For this system, it is possible and convenient to extend the definition of entropy so that it applies to the flow of heat between the open sub-regions, with entropy flux density satisfying $J_{s}=J / T$. This is consistent with the principles of extended thermodynamics [11]. This prescription can be satisfied by an entropy density

$$
v=\rho C_{V} \ln \left(T / T_{s}\right),
$$

where $T_{s}$, inserted for dimensional consistency, is a natural temperature scale such as the uniform equilibrium temperature of a finite region. From the linear heat equation, one easily derives the reactiondiffusion equation

$$
v_{t}=(-J / T)_{x}+k\left(\frac{T_{x}}{T}\right)^{2} .
$$

The entropy source term is non-negative and is zero only for equilibrium states with uniform temperature. The associated total entropy functional $\mathrm{S}$ is the continuous analogue of the discrete Burg entropy [12],

$$
S=\int_{a}^{b} v(x, t) d x
$$

For all non-uniform solutions satisfying zero-flux (perfect insulation) boundary conditions, $\mathrm{S}$ increases with time:

$$
\frac{d S}{d t}=\int_{a}^{b} \frac{\partial v}{\partial t} d x=[J / T]_{a}^{b}+\int_{a}^{b} k\left(\frac{T_{x}}{T}\right)^{2} d x \geq 0 .
$$

A direct connection can be made with information theory since the linear diffusion equation is satisfied also by the probability density function $\mathrm{p}(\mathrm{x}, \mathrm{t})$ of a particle subjected to Brownian motion (e.g. [13]). More generally, the positive solutions $u(x, t)$ of any nonlinear conservation equation on $[a, b] \times \Re^{+}$, with constant integral $U=\int_{a}^{b} u d x$, may be normalised so that they assume the properties of a probability 
density $p=u / U$. As a measure of information content, it is convenient to evaluate the Shannon entropy, which is the expected value of $\log _{2}(1 / p)$ [14]. The Shannon information $\mathrm{S}$ has a simple interpretation as the number of binary digits of accuracy in the measurement of a random variable. It is analogous to an extensive thermodynamic variable, having the desirable feature of additivity. That is, if $S_{1}$ and $S_{2}$ are the entropies of probability densities $p_{1}\left(x_{1}\right)$ and $p_{2}\left(x_{2}\right)$ of independent random variables with values in $\left[a_{1}, b_{1}\right]$ and $\left[a_{2}, b_{2}\right]$, then the entropy of joint probability density $p_{1}\left(x_{1}\right) p_{2}\left(x_{2}\right)$ is $S_{1}+S_{2}$. The discrete Burg entropy, and more generally, linear combinations of the discrete Burg and discrete Shannon entropies [12], also have the additivity property. The continuous Burg entropy is additive after all random variables are normalised to take values in $[0,1]$. In order to consider the information-irreversibility properties of $u(x, t)=U p(x, t)$, it is sufficient to consider the evolution of the quantity $-u \ln (u(x, t))$ whose integral is merely a linear function of the Shannon information,

$$
\int_{a}^{b}-u \ln (u) d x=-U \ln (U)-U \ln 2 \int_{a}^{b} p \log _{2}(p) d x .
$$

Henceforth, $\log$ will refer to the natural logarithm and s will refer to the standard entropy density $-u \log (u)$, where $\mathrm{u}$ is the dependent variable under consideration in the primary transport equation. $\mathrm{S}$ will refer to the standard entropy which is the integral

$$
S=\int_{a}^{b} s d x
$$

For the relationship between Boltzmann's entropy and Shannon's information, we refer to [14].

There are many available choices of Liapunov functionals to indicate irreversibility of the heat equation. With $\mathrm{T}(\mathrm{x}, \mathrm{t})$ satisfying (1), the variable $v=f(T)$ satisfies the reaction-diffusion equation

$$
v_{t}=D v_{x x}-D f^{\prime \prime}(T) T_{x}^{2}
$$

This has a non-trivial non-negative source term if and only if $f^{\prime \prime}(T)<0$. For those cases, the functional

$$
S=\int_{a}^{b} f(T(x, t)) d x
$$

has the property that for all solutions satisfying zero-flux boundary conditions, $d S / d t \geq 0$. Furthermore, $d S / d t=0$ if and only if $\mathrm{T}$ is uniform (thermal equilibrium). This includes the cases $f(T)=\log (T)$ and $f(T)=-T \log (T)$ considered above, as well as the simplest example $f(T)=-T^{2}$. In that case, -S is simply a multiple of the squared $L^{2}$ norm, which is known to decrease because the evolution is contractive. The evolution governed by the linear heat equation is contractive on $L^{2}([a, b])$ since it is of the form

$$
u_{t}=L u,
$$

where $L=\partial_{x}^{2}$, a self-adjoint contraction operator.

The heat equation cannot be derived from an extremal action principle when the Lagrangian density is defined locally (e.g. [15] ). However, viewed as a linear mass transport equation for mass concentration 
$c(x, t)$ of a species of particle, it can be generated in another straightforward way from a notional energy functional

$$
H=\int_{a}^{b} \varepsilon d x
$$

with energy density $\varepsilon=0.5 c^{2}$. Then the chemical potential per unit mass $\mu$ is given by the variational derivative $\delta H / \delta c$, which in this case is simply c. The particle drift velocity $v_{d}$ is then determined by a balance between driving force and linear drag, with resistance coefficient $r$. Hence the mass flux is

$$
J=\rho v_{d}=\frac{-1}{r} \frac{\partial \mu}{\partial x}=-D \frac{\partial c}{\partial x}
$$

with $\mathrm{D}=1 / \mathrm{r}$, constant as in Fick's law. The system is dissipative, since the energy is merely a multiple of the squared $L^{2}$ norm, known to be decreasing in time except when it attains its minimum value. The equations for notional energy density $\varepsilon$ and for entropy density $s=-c \log (c)$ are

$$
\begin{array}{r}
\varepsilon_{t}=D \varepsilon_{x x}-D c_{x}^{2} \\
s_{t}=D s_{x x}+D c_{x}^{2} / c .
\end{array}
$$

Hence, the production rate of entropy is $(1 / \mathrm{T})$ times the dissipation rate of energy. In this sense, $\mathrm{s}$ is the entropy density that is thermodynamically conjugate to energy density $\varepsilon$.

In thermodynamics, an extensive variable $S$ is conjugate to an intensive variable $\mathrm{T}$ if the energy cost in increasing $\mathrm{S}$ is $\delta E=T \delta S$. It is a simple matter to extend this concept to a conjugate triple $(T, s, \varepsilon)$, where $s$ is the locally measured density of $S$ and $\varepsilon$ is the locally measured density of $E$. As the notation suggests, one example is the temperature-entropy-enthalpy triple. Another is the volumepressure-work triple, reminiscent of the generalised displacements that do work against the generalised forces of analytical dynamics. Given a scalar evolution equation with dependent variable $T$, a pair of locally defined smooth functions $(\varepsilon, s)$, constructed from $\mathrm{u}$ and its derivatives, will be said to be an energy-entropy pair if $\varepsilon$ has a source density $-R$ with $R \geq 0$ and not identically 0 , and $s$ has a source density $-\mathrm{R} / \mathrm{T}$. For a closed system at uniform temperature, this agrees with the Clausius definition,

$$
\delta S=\delta E / T
$$

but the definition applies locally to the more general situation of an open sub-region having transport through its boundaries and with T non-uniform. Admittedly, in real problems of non-equilibrium thermodynamics, temperature and entropy may not even be well defined. For the present study of scalar partial differential equations, the dependent variable $\mathrm{T}$ is taken as primitive.

\section{Nonlinear diffusion equations}

In practice, thermal diffusivity may depend appreciably on temperature (e.g. [16]). Similarly, solute transport coefficients may depend on concentration (e.g. [17]). In some applications of parabolic equations, the transport equations may depend also on derivatives of the dependent variable (e.g. [18]). This leads to nonlinear parabolic equations of the general form

$$
u_{t}=\left[-J\left(u, u_{x}\right)\right]_{x} .
$$




\section{Definition}

An evolution PDE is information-irreversible if in any adiabatic evolution, the standard entropy increases monotonically in time and its rate of increase is not identically zero.

This definition, referring only to an integral quantity S, applies also to weak formulations of the PDE. In classical formulations, information-irreversibility is equivalent to $s$ having a non-negative source term that is not identically zero.

The entropy equation, derived from (9), is

$$
s_{t}=([1+\log (u)] J)_{x}-J u_{x} / u
$$

Hence it is easily seen that a second order conservation equation (9) is information-irreversible if and only if the flux is of the form $J=J_{0}(u)$ plus an odd function of $u_{x}$ that is positive when $u_{x}$ is negative.

Since the general second order nonlinear parabolic heat equation takes the same form as (9) (T replacing $\mathrm{u}$ ), we have the direct result that information irreversibility is equivalent to the Clausius form of the second law of thermodynamics, namely that heat must flow from regions of higher temperature to regions of lower temperature. In particular, every second order nonlinear diffusion equation (11) is information-irreversible.

Now consider a notional energy functional

$$
H=\int_{a}^{b} F(u(x, t)) d x
$$

where $\mathrm{F}$ is an arbitrary twice differentiable non-linear function. The chemical potential is $F^{\prime}(u)$ and the flux is $J=-D(u) u_{x}$, where $D(u)=F^{\prime \prime}(u)$. The conservation equation for $u$ is

$$
u_{t}=\left[D(u) u_{x}\right]_{x}
$$

The reaction-diffusion equation for energy density $\varepsilon=F(u)$ is

$$
\varepsilon_{t}=\left[D(u) \varepsilon_{x}\right]_{x}-J^{2} .
$$

Partnered to $\varepsilon$, the entropy density variable, whose production rate is $J^{2} / u$, is

$$
v=-\int^{p} \int^{p_{2}} \frac{D\left(p_{2}\right)}{p_{2}} d p_{2} .
$$

In the special case of constant $\mathrm{D}, v$ is identical to $\mathrm{s}$, a linear function of the Shannon information. Given (11), the reaction-diffusion equation for $\mathrm{s}$ is

$$
s_{t}=\left[D(u) s_{x}\right]_{x}-J \frac{u_{x}}{u} .
$$

$\mathrm{s}$ is always thermodynamically partnered to the density $e=0.5 u^{2}$, which satisfies the equation

$$
e_{t}=\left[D(u) e_{x}\right]_{x}+J u_{x}
$$


For positive solutions $u(x, t)$, both the standard entropy and the partnered entropy increase in time.

More general Liapunov functionals arise when we re-express a time evolution equation in terms of a new set of independent variables. For example, after using a similarity invariant as the spatial variable, the Barenblatt similarity solutions of the porous medium equations with power-law $D(u)$ may be viewed as stable equilibrium states of minimized generalized entropy [19, 20].

\section{Fourth Order Diffusion}

Unlike second order diffusion equations, fourth order equations do not in general satisfy any known maximum principle. Even under the simplest time-independent linear boundary conditions, evolving solutions tend to generate additional extrema from smooth initial conditions. Therefore, we are motivated to investigate the entropy behaviour predicted by these equations.

\subsection{Case of constant diffusion coefficient}

The simplest example is the linear equation with constant coefficients

$$
u_{t}=-u_{x x x x}
$$

which is generated by the energy functional [21]: $H=\int_{a}^{b} u_{x}^{2} d x$. The minus sign in (14) is required to make this a dissipative equation on $L^{2}((a, b))$,

$$
\left(u^{2}\right)_{t}+\left[\left(u^{2}\right)_{x x x}-4\left(u_{x}^{2}\right)\right]_{x}=-2 u_{x x}^{2} \leq 0 .
$$

It is convenient to regard Equation (14) as having diffusion coefficient 1, rather than -1. However this equation is not information-irreversible:

$$
\begin{array}{r}
s_{t}=(1+\log (u)) u_{x x x x} \\
=\left((1+\log (u)) u_{x x x}\right)_{x}-u^{-1} u_{x} u_{x x x} .
\end{array}
$$

The current-source decomposition of a quantity $-J_{x}+R$ as in (16) is not unique. However we shall now proceed to identify a quantity that may be regarded as the irreducible source term. Given an equation

$$
s_{t}=-J_{x}+R^{(n)}
$$

with an order $\mathrm{n}$ remainder term $R^{(n)}$ that is quasilinear, the equation may be re-expressed as

$$
s_{t}=-\left[J+J^{(n-1)}\right]_{x}+R^{(n-1)}
$$

with $R^{(n-1)}$ and $J^{(n-1)}$ of order n-1. For example, if $R=h\left(u_{x}\right) u_{x x}+j\left(u_{x}\right)$, then $R=\left[H\left(u_{x}\right)\right]_{x}+j\left(u_{x}\right)$, where $H^{\prime}=h$. If $R^{(n-j)}$ is a meromorphic function in $\mathrm{u}$ and its derivatives, then as shown in Appendix A, all quasilinear terms can be removed by iterations of this procedure to achieve a fully nonlinear polynomial remainder in which each term is fully nonlinear. This irreducible polynomial remainder is unique and we shall regard it as the irreducible source term. 
By this algorithm, (16) is recast, with an irreducible source term, as

$$
s_{t}=\left((1+\log (u)) u_{x x x}-u^{-1} u_{x} u_{x x}-\frac{1}{3} u^{-2} u_{x}^{3}\right)_{x}-\frac{2}{3} u^{-3} u_{x}^{4}+u^{-1} u_{x x}^{2} .
$$

The irreducible source term is indefinite in sign, so the 4th order diffusion equation is not informationirreversible. For equations such as (14), additional structure in the form of new extrema may evolve from simpler initial conditions in a Cauchy initial value problem. For example, from Gaussian initial conditions, the solution may evolve towards a stable self similar point source solution

$$
u=t^{-1 / 4} f\left(x t^{-1 / 4}\right)
$$

that has an infinite number of extrema [22]. This is shown in Figure 1.

Figure 1. Solution of fourth order linear diffusion equation with constant diffusion coefficient 1 , Gaussian initial condition and boundary conditions of zero flux and zero gradient far from the origin. Solution at time $t=0.2$ was evaluated by the library PDE solver of Matlab [27].

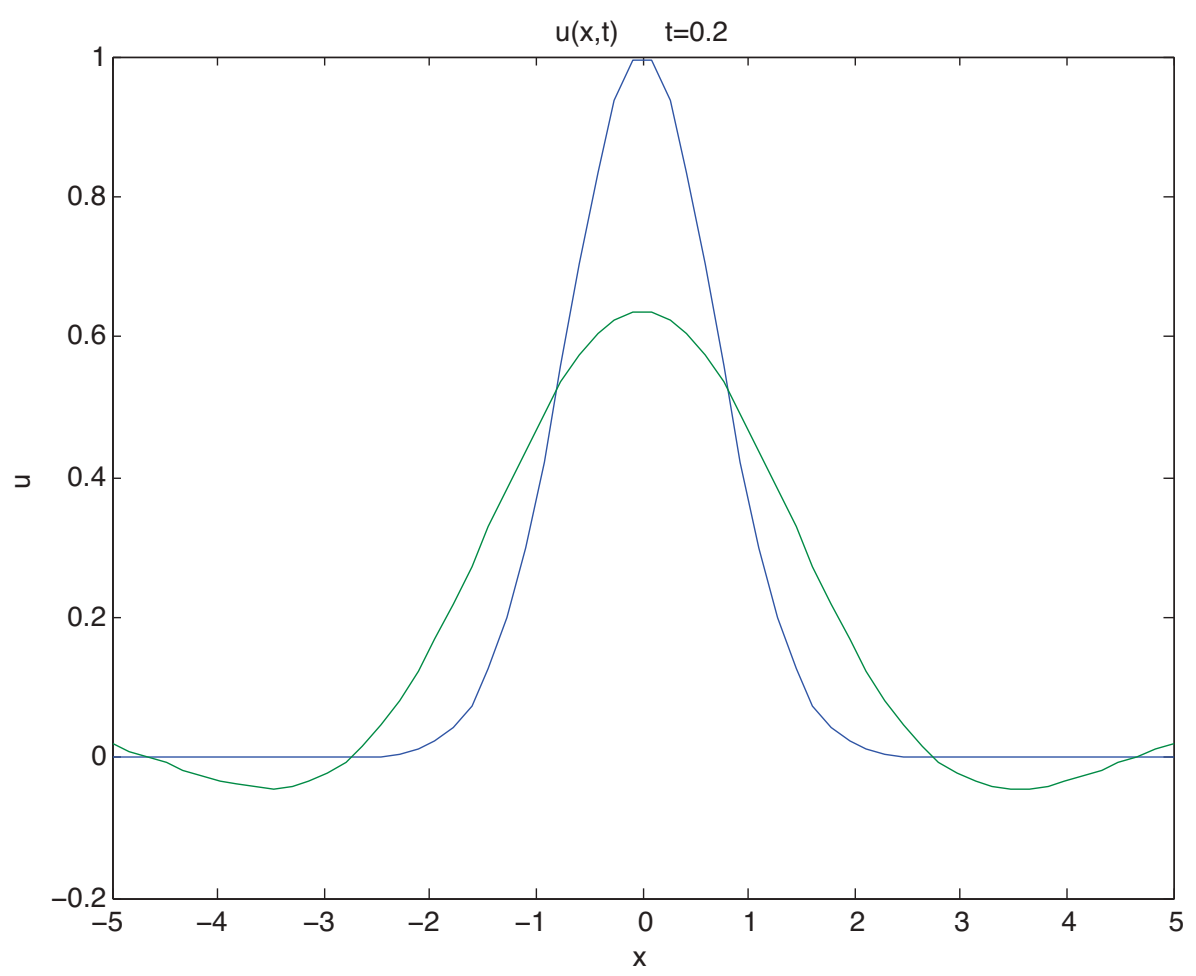

\subsection{Case of nonlinear diffusion coefficient $G(u)$}

We now raise the question of whether any 4 th order conservation equations are in fact, informationirreversible. First we consider the class with a single nonlinear diffusion coefficient $G(u)$

$$
u_{t}=\left(-G(u) u_{x x x}\right)_{x} .
$$


For this class, the reaction-diffusion equation for entropy density is

$$
s_{t}=\left[[1+\log (u)] G(u) u_{x x x}-\frac{G(u)}{u} u_{x} u_{x x}+\frac{1}{3}\left(\frac{G(u)}{u}\right)^{\prime} u_{x}^{3}\right]_{x}-\frac{1}{3}\left(\frac{G(u)}{u}\right)^{\prime \prime} u_{x}^{4}+\frac{G(u)}{u} u_{x x}^{2} .
$$

Hence, (18) is information-irreversible if and only if $\left(u^{-1} G(u)\right)^{\prime \prime} \leq 0$ and $u^{-1} G(u)>0$. For positive solutions, this includes the power laws $G(u)=u^{n}$, with $1 \leq n \leq 2$. Known positivity results of weak solutions to these and other 4th order equations, which are limits of solutions to modified non-degenerate equations, are summarised by Beretta et al [23]. For this purpose, based on earlier analysis by Bernis and Friedman [24], Ulusoy [21] used bounds on the rate of decrease of entropy-like Liapunov functionals.

In Figures 2 and 3, we depict the solution for the fourth order nonlinear diffusion equations with $G(u)=u$ and $G(u)=u^{2}$ respectively, with Gaussian initial condition and boundary conditions of zero gradient and zero flux far from the origin. In these cases of degenerate nonlinear diffusion, the solution approaches the stable point-source weak solution that is known to have compact support and continuous first derivative $[22,23]$.

Figure 2. Solution of fourth order nonlinear diffusion equation with diffusion coefficient $u$, Gaussian initial condition and boundary conditions of zero flux and zero gradient far from the origin. Solution at times $\mathrm{t}=0.5,4.0$ was evaluated by the library PDE solver of Matlab.

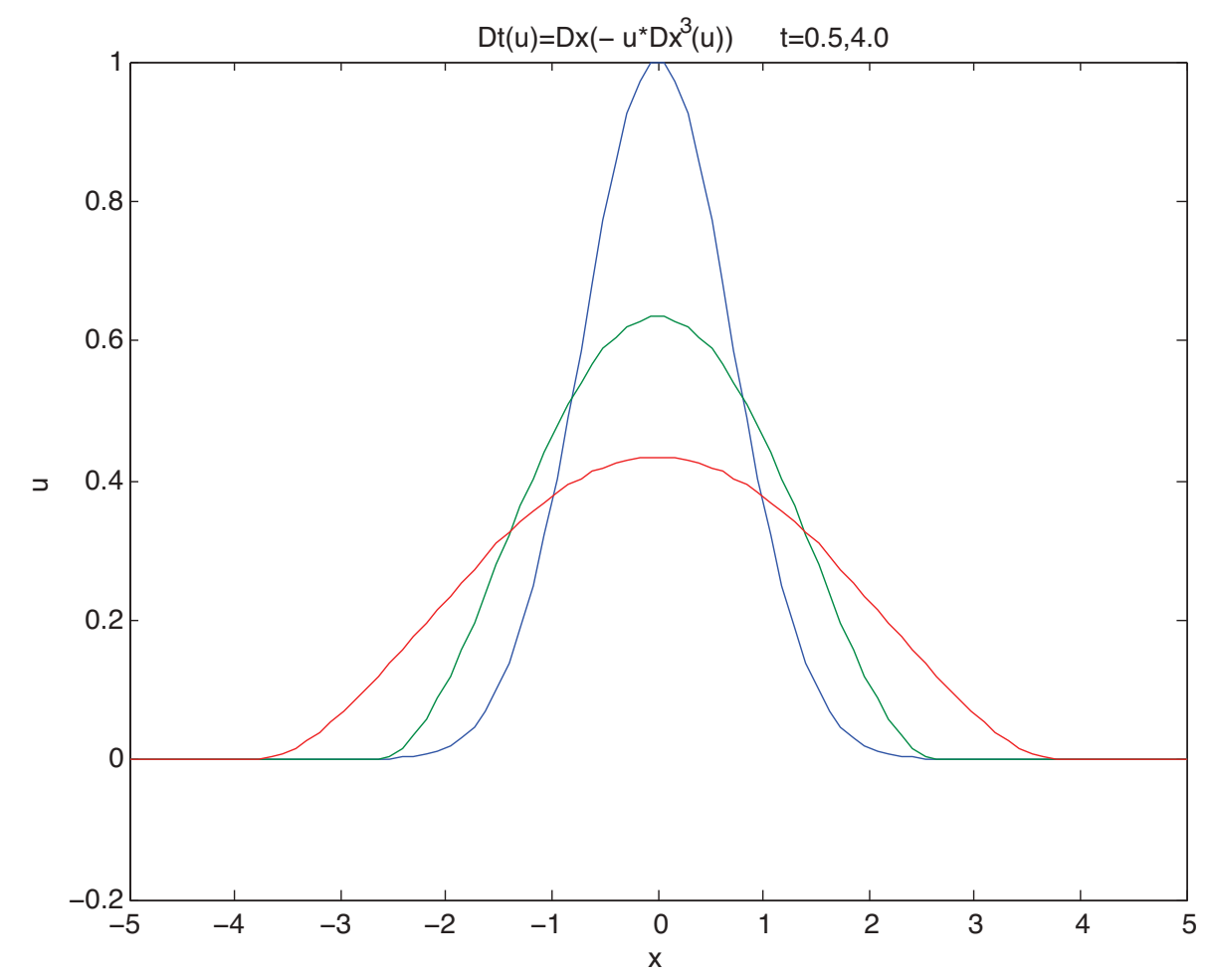


Figure 3. Solution of fourth order nonlinear diffusion equation with diffusion coefficient $u^{2}$, Gaussian initial condition and boundary conditions of zero flux and zero gradient far from the origin. Solution at times $\mathrm{t}=0.5,4.0$ was evaluated by the library PDE solver of Matlab.

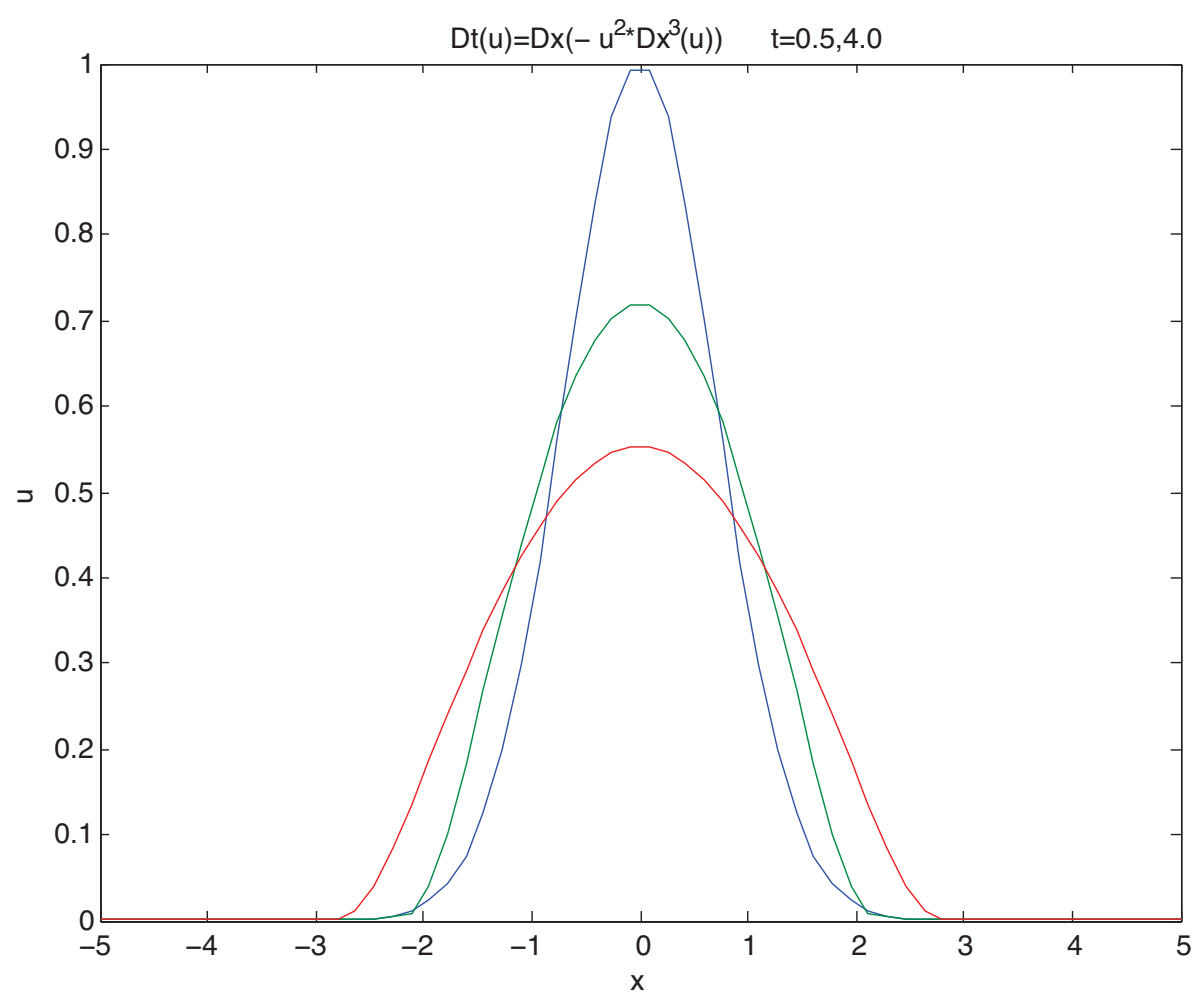

\subsection{Semilinear conservation equations}

Next we ask if the constant-coefficient 4th order diffusion equation (14) can be modified by lower order flux terms to make it an information-irreversible conservation equation. Consider an equation of the general form

$$
u_{t}=-\left[u_{x x x}+h\left(u, u_{x}, u_{x x}\right)\right]_{x} .
$$

This implies an equation for the standard entropy density

$$
s_{t}=\left[(1+\log (u)) u_{x x x}-\frac{u_{x}}{u} u_{x x}-\frac{1}{3} \frac{u_{x}^{3}}{u^{2}}+(1+\log (u)) h\right]_{x}+\frac{u_{x x}^{2}}{u}-\frac{2}{3} \frac{u_{x}^{4}}{u^{3}}-\frac{u_{x}}{u} h .
$$

Now we may choose $h$ so that further terms are generated in the irreducible source to cancel the negative contribution $-(2 / 3) u^{-3} u_{x}^{4}$. The necessary components of $\mathrm{h}$ that can generate such terms are of the general form

$$
h=a u^{-1} u_{x} u_{x x}+b u^{-2} u_{x}^{3} .
$$

With $h$ taking this general form, the entropy equation is modified to

$$
\begin{array}{r}
s_{t}=-J_{x}+R \\
\text { with }-J=(1+\log (u)) u_{x x x}-\frac{u_{x}}{u} u_{x x}-\frac{1}{3} \frac{u_{x}^{3}}{u^{2}}+(1+\log (u)) h-\frac{a}{3} \frac{u_{x}^{3}}{u^{2}}
\end{array}
$$




$$
\text { and } R=\frac{u_{x x}^{2}}{u}-\left[\frac{2}{3}(1+a)+b\right] \frac{u_{x}^{4}}{u^{3}} .
$$

Hence, we achieve a non-trivial non-negative entropy source term when

$$
b \leq \frac{-2}{3}(a+1) .
$$

For example, when $\mathrm{a}=-1$ and $\mathrm{b}=0$, the entropy source density is $u_{x x}^{2} / u$. In that case, the governing conservation equation for $\mathrm{u}$ is

$$
u_{t}=-\left[u_{x x x}-u^{-1} u_{x} u_{x x}\right]_{x} .
$$

This is closely related to the Derrida-Lebowitz-Speer-Spohn (DLSS) equation

$$
u_{t}=-u_{x x x x}+\left(u^{-1} u_{x}^{2}\right)_{x x}
$$

which also has monotonic Shannon entropy, and which preserves positivity[25]. After choice of similarity coordinates, the DLSS equation (26) is seen to have a stable similarity solution that is the equilibrium state of a modified Shannon entropy functional [26].

In Figure 4, we plot the solution of (25) with Gaussian initial conditions. The solution evidences smoothing properties, it maintains positivity and unlike the solutions of degenerate diffusion equations, there is no known stable nontrivial solution with compact support. Its behaviour resembles that of a standard point source solution of the classical second order diffusion equation but the actual point-source solution broadens in proportion to $t^{1 / 4}$ rather than $t^{1 / 2}$.

\subsection{Case of nonlinear diffusion coefficient $G\left(u_{x}\right)$}

As a final example of an information-irreversible equation, we consider equations of the form

$$
u_{t}=-\left[G\left(u_{x}\right) u_{x x x}\right]_{x} .
$$

We find that the only power law function that gives rise to increasing entropy is

$$
G\left(u_{x}\right)=-u_{x}^{-2}
$$

\subsection{Mullins surface diffusion equation}

Mullins [3] derived the equation for surface diffusion on metals as a fourth order curvature-driven flow,

$$
y_{t}=-\left[\frac{1}{\sqrt{1+y_{x}^{2}}}\left(\frac{y_{x x}}{\sqrt{1+y_{x}^{2}}}\right)_{x}\right]_{x} .
$$

This implies a reaction-diffusion equation for the standard entropy density that has an irreducible source term

$$
\frac{-2}{y} \frac{y_{x}^{2}}{\left(1+y_{x}^{2}\right)^{3}}+\frac{2}{y^{3}}\left[\frac{y_{x}^{2}}{1+y_{x}^{2}}-\frac{1}{y^{2}} y_{x} \arctan \left(y_{x}\right)\right] \text {, }
$$

which may take either sign. Hence, the surface diffusion equation is not information-irreversible. This is consistent with the known tendency of the surface diffusion equation to generate ripples from smooth initial conditions, given simple boundary conditions $[4,5]$. 
Figure 4. Solution of fourth order semilinear diffusion equation (25), Gaussian initial condition and boundary conditions of zero flux and zero gradient far from the origin. Solution at times $\mathrm{t}=0.2,0.8$ was evaluated by the library PDE solver of Matlab.

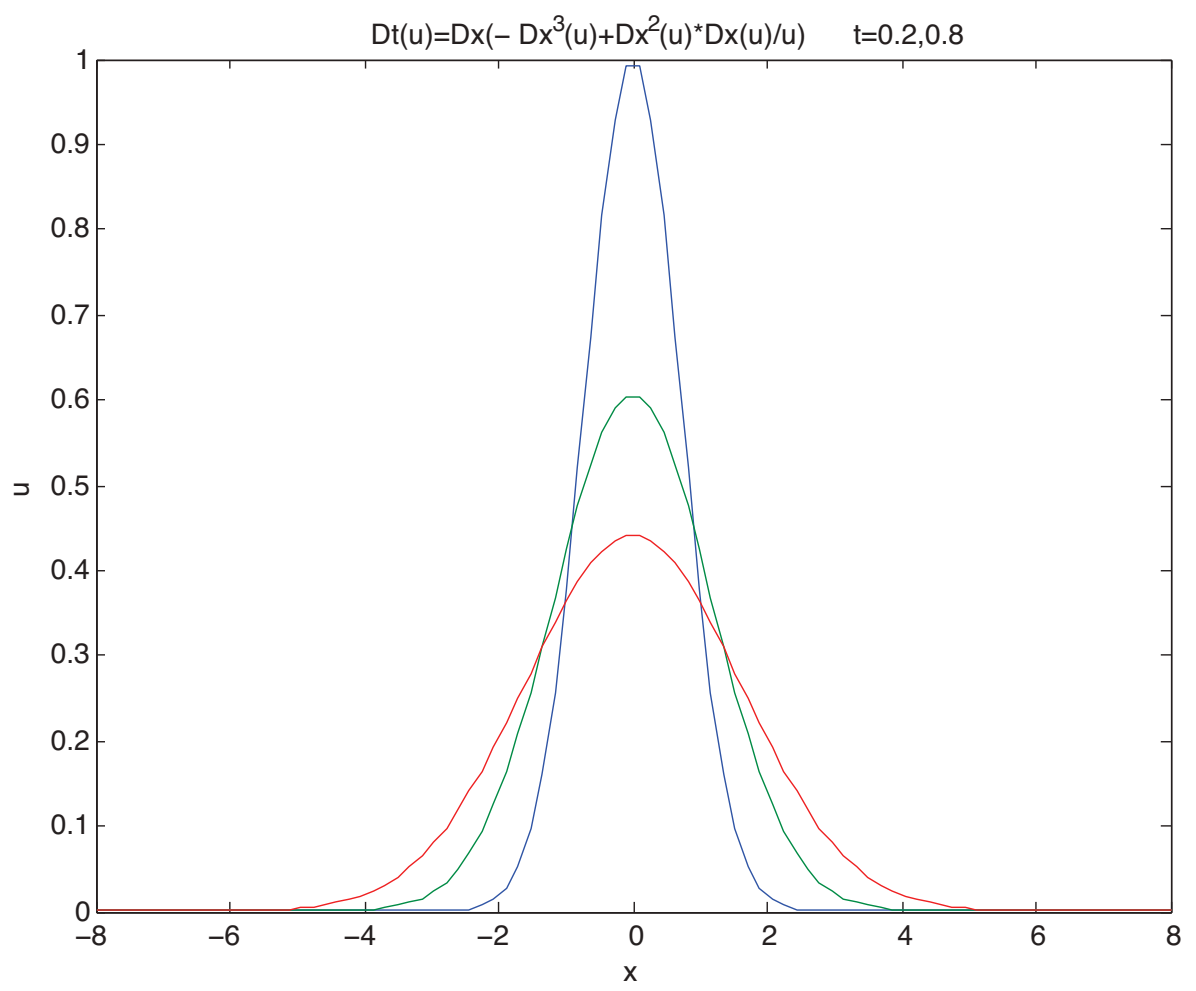

\section{Conclusion}

A partial differential equation in conservation form, subject to zero-flux boundary conditions, conserves mass and so its finite-mass solutions may be normalized to take the form of an evolving probability density. From this, the key concept of information irreversibility follows naturally. Irreversibility is traditionally highlighted by monotonicity of a global integral quantity such as the total entropy functional. However, for diagnostic purposes it is more direct to consider a local form of entropy density. From the PDE governing the probability density, we may construct a reaction-diffusion equation for the Shannon entropy density. However, since the current-source decomposition of a reaction-diffusion equation is non-unique, we have had to develop a second key concept, that of an irreducible source term, which is uniquely defined at least for polynomial source terms and which seems to be practically useful as a diagnostic tool. The information irreversibility of the PDE may be analysed directly from the sign of the irreducible fully nonlinear local source term of the entropy density. All second order diffusion equations are information-irreversible. Compared to second order diffusion equations, the entropy behaviour of fourth order equations is quite different. The familiar fourth order diffusion equations that arise in practical applications are not information-irreversible. Contrary to an impression that might easily be formed from the familiar cases, there are some classes of closely related fourth order diffusion equations that are in fact information-irreversible. Information irreversibility seems to be associated with regular smoothing behaviour of solutions, as depicted for example in Figure 4. As far as the author is aware, 
such regular behaviour has not been observed before in the solutions of fourth order evolution equations.

A proof of the uniqueness of the irreducible source term for general functional forms of a reactiondiffusion equation, is given here for the case when the nominal source term is a meromorphic function of the dependent variable and its spatial derivatives. Generally, a monotonic decrease of information is associated with smoothing of ripples. It remains to be seen whether a form of maximum principle might be valid for the restricted class of fourth order nonlinear diffusion equations that obey information irreversibility. In all cases when we have established information-irreversibility, a numerical solution with Gaussian initial conditions maintains positivity and it does not generate new extrema. Conversely, when information is reversible, the solutions generate extrema and they lose positivity. With this limited but suggestive evidence, we conjecture that for zero-flux boundary value problems and positive initial conditions, information-irreversibility implies positivity. It remains to be seen whether additional conditions need to be added before such a conjecture could be established as a true statement.

During the above investigation, we have naturally been led to the concept of a partner entropy that is conjugate to a notional energy density that generates a governing nonlinear PDE. This generalizes the Shannon entropy since the latter is the partner entropy to an energy that generates linear diffusion. However, it is not known if the partner entropy has any use beyond the conceptual. Since the thermodynamic conjugacy of temperature and entropy variables is traditionally defined through an expression for work done, a relation among temperature, entropy and energy variables, it is natural to define a conjugate temperature-entropy-energy triple. One could extend this idea further by applying the Legendre transform of enthalpy with respect to entropy to construct a conjugate temperature-entropy-enthalpy-free energy quadruple but this serves little purpose in the present paper.

In principle, one could define an entropy density even from solutions of PDEs that cannot be expressed in conservation form. However, we have not investigated whether such a concept has any use.

\section{Acknowledgements}

The author is grateful to John Schutz, Mary Pugh and Tom Witelski for useful comments and for alerting him to relevant published material.

\section{References}

1. Schwartz, L.W.; Roy, R.V. Theoretical and numerical results for spin coating of viscous liquids. Phys. Fluids 2004, 16(3), 569-584.

2. Myers, T. G. Thin films with high surface tension. SIAM Review 1998, 40(3), 441-462.

3. Mullins, W. Theory of thermal grooving. J. Appl. Phys. 1957, 28(3), 333-339.

4. Tritscher, P.; Broadbridge, P. Grain boundary grooving by surface diffusion: an analytic nonlinear model for a symmetric groove. Proc. Roy. Soc. London A 1995, 450, 569-587.

5. Tritscher, P. An integrable fourth-order nonlinear evolution equation applied to surface redistribution due to capillarity. J. Austral. Math. Soc. Ser. B 1997, 38( 4), 518-541.

6. Allen, S.M; Cahn, J.W. A microscopic theory for antiphase boundary motion and its application to antiphase domain coarsening. Acta Metall. 1979 27, 1085-1095. 
7. Rubinstein, J.; Sternberg, P.; Keller, J.B. Fast reaction, slow diffusion and curve shortening. SIAM J. Appl. Math. 1989, 49, 116-133.

8. Bernoff, A.J.; Bertozzi, A. L. Singularities in a modified Kuramoto-Sivashinsky equation describing interface motion for phase transition. Physica D 1995, 85, 375-404.

9. Caginalp, G. The dynamics of a conserved phase field system: Stefan-like, Hele-Shaw, and CahnHilliard models as asymptotic limits. IMA J. Appl. Math. 1990, 44(1), 77-94.

10. Lieb, E.H; Yngvason, J. The mathematics of the second law of thermodynamics. Geom. Funct. Anal. 2000, Special Issue: Part 1 Sp. Iss., 509-526.

11. Müller, I. Entropy in Nonequilibrium. In Entropy; Greven, A.; Keller, G.; Warnecke, G. Eds.; Princeton University Press: Princeton, 2003; pp. 79-104.

12. Gorban, A. N.; Karlin, I. V. Family of additive entropy functions out of thermodynamic limit. Phys. Rev. E. 2003, 67,016104,1-10.

13. Kramer, P. R. Brownian Motion. In Encyclopedia of Nonlinear Science; Scott, A. (Ed.) Routledge: New York, 2005; pp. 78-80.

14. Jaynes, E.T. Information theory and statistical mechanics. Phys. Rev. 1957, 106(4), 620-630.

15. Taflin, E. Analytic linearization, Hamiltonian formalism, and infinite sequences of constants of motion for the Burgers equation. Phys. Rev. Lett. 1981, 47(20), 1425-1428.

16. Y. S. Touloukian, Y.S. (Ed.) Thermophysical Properties of Matter Vol. 10 Thermal Diffusivity. Plenum, New York, 1974.

17. Bardow, A.; Goke, V.; Koss, H.J.; Lucas, K.; Marquardt, W. Concentration-dependent diffusion coefficients from a single experiment using model-based Raman spectroscopy. Fluid Phase Equilibria 2005, 228 Spec. Issue , 357-366.

18. Broadbridge, P. Exact solvability of the Mullins nonlinear diffusion model of groove development. J. Math. Phys. 1989, 30(7),1648-1652.

19. Newman, W. I. A Lyapunov functional for the evolution of solutions to the porous medium equation to self-similarity, I. J. Math. Phys. 1984, 25, 3120-3123.

20. Witelski, T. P.; Bernoff, A. J. Self-similar asymptotics for linear and nonlinear diffusion equations Studies Appl. Math. 1998, 100,153-193.

21. Ulusoy, S. A new family of higher order nonlinear degenerate parabolic equations. Nonlinearity 2007, 20, 685-712.

22. Smyth, N. F.; Hill, J. M. High-order nonlinear diffusion. IMA J. Appl. Math. 1988, 40(2), 73-86.

23. Beretta, E.; Bertsch, M.; Dal Passo, R. Nonnegative solutions of a fourth-order nonlinear degenerate parabolic equation. Arch. Ration. Mech. Anal. 1995, 129, 175-200.

24. Bernis, F.; Friedman, A. Higher order nonlinear degenerate parabolic equations. J. Diff. Eqns 1990, 83, 179-206.

25. Bleher, P.; Lebowitz, J.; Speer, E. Existence and positivity of solutions of a fourth-order nonlinear PDE describing interface fluctuations. Commun. Pure Appl. Math. 1994, 47, 923-942.

26. Carrillo, J. A.; Toscani, G. Long-time asymptotics for strong solutions of the thin film equation. Commun. Math. Phys. 2002, 225, 551-571.

27. The MathWorks inc. Matlab Users' Guide 2006. 


\section{Appendix A: Uniqueness of irreducible source term}

Suppose that $A=-J_{x}+R$, where $\mathrm{R}$ is a Laurent expansion in $\mathrm{u}$ and its derivatives $u_{n}=\partial_{x}^{n} u$. If any term $R^{(n)}$ of the expansion is quasilinear and of order $\mathrm{n}$,

$$
R^{(n)}=h(x) u^{p 0} u_{1}^{p 1} u_{2}^{p 2} \ldots u_{n-1}^{p_{n-1}} u_{n}
$$

then

$$
R^{(n)}=\left[h(x) u^{p 0} u_{1}^{p 1} u_{2}^{p 2} \ldots u_{n-2}^{p_{n-2}} \int u_{n-1}^{p_{n-1}} d u_{n-1}\right]_{x}-\left[h(x) u^{p 0} u_{1}^{p 1} \ldots u_{n-2}^{p_{n-2}}\right]_{x} \int u_{n-1}^{p_{n-1}} d u_{n-1}
$$

which is of the form $-J_{x}^{(n-1)}+R^{(n-1)}$, where the residual source term is of order $n-1$. Hence we may absorb all quasilinear terms into the derivative current $-J_{x}$. Without loss of generality, we may assume that all terms in the Laurent expansion for R are fully nonlinear. Suppose that we have two equivalent expressions

$$
-J_{x}+R=-I_{x}+P
$$

where $\mathrm{R}$ and $\mathrm{P}$ are different Laurent series in $\mathrm{u}$ and its derivatives, with all terms of $\mathrm{R}$ and $\mathrm{P}$ fully nonlinear. Then

$$
P-R=[I-J]_{x}
$$

However, a total x-derivative $\left[J\left(x, u, u_{1}, \ldots . u_{m}\right)\right]_{x}$ must have quasilinear terms $u_{m+1} J_{u_{m}}$. The fully nonlinear expression P-R cannot be expressed as a total $\mathrm{x}$-derivative. This contradicts the assumption that $\mathrm{P}-\mathrm{R}$ is non-zero. Therefore $\mathrm{R}$ is unique.

(c) 2008 by the authors; licensee Molecular Diversity Preservation International, Basel, Switzerland. This article is an open-access article distributed under the terms and conditions of the Creative Commons Attribution license (http://creativecommons.org/licenses/by/3.0/). 